AJHSE Vol: 1 (2): 52-65, 2020

Article Ref. No.: AJHSE010205

Accepted Date: October 01, 2020

(C) 2020. CC License 4.0

www.ajhse.org

CrossMark

$\leftarrow$ click for updates
African Journal of Health, Safety and Environment

An official publication of the

Applied Environmental Bioscience and Public Health Research Group

University of Benin, Benin City, Nigeria

Open Access | Bi-annual | Peer-reviewed | International ISSN (Online): 2695-1819 | ISSN (Print): 2695-2386

\title{
EXPLORING AWARENESS AND ATTITUDES OF NURSES TOWARDS HERBAL MEDICINE USE DURING PREGNANCY AND BREASTFEEDING
}

\author{
${ }^{1}$ Alharbi R., ${ }^{1}$ Abdulkhaleg N. and ${ }^{* 2}$ Orabi A. M. \\ ${ }^{1, * 2}$ College of Nursing, King Saud Bin Abdul-Aziz University for Health Sciences, Saudi Arabia, Jeddah \\ ${ }^{1}$ Faculty of Nursing, Cairo University, Cairo, Egypt. \\ *Corresponding Author Email: orabia@ksau-hs.edu.sa Phone: +966+966531323879
}

\begin{abstract}
$\mathrm{H}$

erbal medicine is one of the popular complementary and alternative medicine (CAM) therapies used by women. The aim of the present study was to explore awareness and attitudes of nurses

towards herbal medicine use during pregnancy and breastfeeding. A descriptive design was used to collect data from all nurses working at maternity units, King Khalid Hospital, Jeddah. Data were collected using a questionnaire designed by the investigators on socio-demographic background, awareness, and attitudes regarding herbal medicine use during pregnancy and breastfeeding. More than two thirds of the participants heard about herbal medicine and knew about its use by women during pregnancy and breastfeeding ( $88.6 \%$ and $68.6 \%$ respectively). However, more than half of them were not aware of its benefits or harms (55.7\% and 65.7\%). Half of them were familiar to and aware of the effects of ginger (55.7\%) and garlic (51.4\%). However, most of them were unfamiliar of myrrh (78.6\%), fenugreek (71.4\%), and fennel (68.6\%). Participants had neutral and positive attitudes towards herbal medicine use during pregnancy and breastfeeding (43\% and 57\% respectively). Statistically significant correlations were found between attitudes towards herbal medicine and nurses' age and number of children $(\mathrm{P}=0.03)$. Although nurses knew about herbal medicine use by women during pregnancy and breastfeeding and had favorable attitudes towards it, more than half of them need to be aware of its benefits or harms.
\end{abstract}

Keywords: attitudes, awareness, breastfeeding, herbal medicine, nurses, pregnancy

LICENSE: This article by African Journal of Health, Safety and Environment (AJHSE)is licensed and published under the Creative Commons Attribution License 4.0 International License, which permits unrestricted use, distribution, and reproduction in any medium, provided this article is duly cited.

COPYRIGHT: The Author(s) completely retain the copyright of this published article.

OPEN ACCESS: The Author(s) approves that this article remains permanently online in the open access (OA) mode.

QA: This Article is published in line with "COPE (Committee on Publication Ethics) and PIE (Publication Integrity \& Ethics)". 


\section{INTRODUCTION}

Complementary and alternative medicine (CAM) is widely used in many countries although its safety and effectiveness are still uncertain. CAM is a group of healthcare practices and products that are not part of traditional medicine. Typically, it is classified into alternative medical therapy such as traditional Chinese medicine; mind-body therapy as meditation, prayer, and healing or support groups; biologically based therapy as herbs, dietary supplements; manipulation and body-based therapies as massage, chiropractic or osteopathy and energy therapies (National Center for Complementary and Alternative Medicine, 2011). Estimates revealed that, 52\%, 68.5\%, 57.1\%, and 50.7\% of pregnant women used CAM therapy in Australia, USA, UK, and Germany respectively (Frawley et al., 2013; Strouss et al., 2014; Hall and Jolly, 2014; Kalder et al., 2011). Interestingly, CAM was reported as the most prevalent treatment option around the world for obstetrical problems (Kalder et al., 2011). Reported reasons were its availability while lack of access to health care services (AlBedah et al., 2010), having control in care, fear of side effects of medical interventions especially during pregnancy (Mitchell and Mcclean, 2014).

Concerning the picture of non-traditional therapies in Kingdom of Saudi Arabia, the commonly used CAM therapies were prophetic medicine, including prayer, honey, medical herbs, Hijama, nutritional supplements, cauterization, camel milk, massage and acupuncture $(90.5 \%, 85 \%, 76.9 \%, 70.6 \%, 61.4 \%, 55.9 \%, 52.5 \%, 61.8 \%$ and $55 \%$ respectively) (AlBedah et al., 2012). At the same context, studies conducted in Riyadh region showed that, threequarters of participants had used CAM and less than half of them consulted CAM provider (Abdullah et al., 2012).

Herbal medicine is one of the poplar CAM therapies used by women and its use is increasing globally, specifically during pregnancy and breastfeeding (Hall et al., 2011). In the Middle East, a wide range of pregnant women were using herbal medicine (22.3\%-82.3\%) and the prevalent herbs were ginger, thyme, chamomile, sage, aniseed, peppermint, fenugreek, and green tea that were used for treatment of gastrointestinal disorders, cold and flu symptoms (Lisha and Nisha, 2015). Most of the women used these products during first trimester without informing their physician. However, they based their decisions on personal experience or recommendations of family or friend (Frawley et al., 2014).

In Saudi Arabia, many foreign non-Moslem nurses work in the obstetric units. At the same time, Saudi childbearing women expect orientation and commitment to Muslim believes and cultural practices during pregnancy, postpartum and breastfeeding. Additionally, nursing interventions should be culturally sensitive to promote positive outcomes. So, nurses need to have culture-specific knowledge and skills to be able to provide care for women from different culture. In this perspective, a previous study concluded that, $86 \%$ of the non-Moslem nurses desired to have lectures on Saudi culture especially topics related to antenatal care, labor and breastfeeding practices. However, another study revealed that, $14 \%$ of nurses asked pregnant women about herbal products and additionally, $86 \%$ of the nurses had no knowledge about the herbs commonly used by Muslim women. Furthermore, $8 \%$ of physicians in Riyadh had training on CAM and 14.2\% referred their patients to CAM practitioners (Sidumo et al., 2010).

Notably, use of herbal medicine is prevalent among pregnant and breastfeeding women in the region with an incomprehensive picture in Saudi Arabia (Sidumo et al., 2010). Moreover, it will be a major challenge for health care professionals if they are not informed with the use (Adams, 2011). However, herbs should be appropriately prescribed and used during these risk periods to prevent interaction with prescribed medications and resulting fetal or neonatal 
complications (Holst et al., 2008; Samuel et al., 2010; Al-Rowais et al., 2012; Alrawi and Fetters, 2012; Abdullah et al., 2013). In the light of restricted studies investigating attitudes towards CAM use and conditions indicating its recommendation for pregnant women (Samuel et al., 2010) in addition to variable responses of previous studies regarding the safety and efficacy of some types, attitudes, and education of health care providers regarding their use require attention. Taking into account, conclusions from previous studies which emphasized the need to assess awareness and attitudes of health care providers regarding the commonly used CAM practices in the region for better outcomes achievement (Al-Rowais et al., 2012). In line with this theme, the current study aimed at exploring awareness and attitudes of nurses in Jeddah region, Saudi Arabia, towards herbal medicine use during pregnancy and breastfeeding. The study aimed at exploring awareness and attitudes of nurses towards herbal medicine use during pregnancy and breastfeeding.

\section{MATERIALS AND METHODS}

\section{DATA COLLECTION, TOOL AND PROCEDURE}

Data were collected at King Khalid Hospital, Jeddah, from four units/wards: antenatal and gynaecology ward, postnatal ward, labor and delivery unit, and maternal and neonatal monitoring unit. Seventy nurses representing nearly all nurses working in the study settings that consented to participate in the study were recruited using convenience sampling technique. Only, nurses, who were working in KSA for 2 years or more, were included. A descriptive exploratory design was used to understand phenomena of interest.

An English questionnaire was designed by the researchers after extensive literature review. and completed by the participants. The self-administered questionnaire encompasses three sections (57 items). First section elicited information on sociodemographic background. Second section assessed awareness of herbal medicine use during pregnancy and breastfeeding. Third section investigated attitudes of the participants towards herbal medicine use during pregnancy and breastfeeding, it included 30 statements on 5 points Likert scale starting from strongly agree (5) to strongly disagree (1) with a total score out of 150 . The scoring system used to categorize the attitudes was as the following: a score $<50$ indicates negative attitude (0-33.3\%), a score of 51 to 100 indicates neutral attitude (33.34$66.6 \%)$ and a score $>100$ indicates positive attitude $(66.7-100 \%)$.

The researchers distributed the questionnaire, followed up its completion and finally collected it. Content validity of the questionnaire was confirmed by a panel of nursing experts in paediatrics, community health and research who assessed its relevancy and appropriateness to the current study aims then necessary changes were done. Reliability was confirmed through Cronbach's Alpha testing (0.89). A pilot study was conducted by introducing the questionnaire to 9 nurses ( $10 \%$ of the participants) who met the inclusion criteria, to assess clarity of the items and feasibility of the study, no modification was needed. Respondents who were recruited for pilot study were included in the whole study participants. 


\section{DATA ANALYSIS}

Data were coded, entered, and analyzed using the Statistical Package for Social Sciences (SPSS) version 18 for Windows. Data was presented using descriptive statistics including frequencies, percent, means and standard deviations. Also, inferential statistics were used to examine the significance of comparison and correlation between the study variables. A $p<0.05$ was used as a significance level.

\section{ETHICAL CONSIDERATION AND STUDY LIMITATIONS}

The study was conducted after the proposal had been reviewed and approved by the College of Nursing Research Unit, the Institutional Review Board (IRB) at King Khalid Hospital and King Abdullah International Medical Research Center (KAIMRC), Jeddah, KSA. Then, the researchers explained the aim and nature of the study to all the respondents who met the inclusion criteria to obtain their consent to participate in the study. In addition, the researchers emphasized that participation in the study is voluntary and confidentiality will be maintained.

Although the current study provided some insight on nurses' awareness and attitudes towards herbal medicine use during pregnancy and breastfeeding, small ample size and convenient sampling technique were of its limitations that might affect generalizability of the revealed findings. Considering that, number of participants was less than estimated because some of the eligible nurses did not participate because of their limited time.

\section{RESULTS}

Considering the socio-demographic characteristics of the participants, age ranged between 22 and 62 with a mean of $35.57 \pm 9.13$ years, two thirds $(65.7 \%)$ of them had a bachelor degree, and a little above half of them (55.7\%) were married. The mean number of children was $1.1 \pm 1.4$ while, the mean years of work experience in KSA was $7.7 \pm 6.3$. Majority of the participants (80\%) were non-Saudi (34.3\% Philippines, 28.6\% Malaysian, $11.4 \%$ Indian, Jordanian $2.9 \%, 1.4 \%$ British \& $1.4 \%$ Slovakian) (Table 1). 
Table 1: Sociodemographic characteristics of the participants.

\begin{tabular}{|c|c|c|c|}
\hline Item & Distribution & Frequency $(\mathrm{n}=70)$ & Percentage $(\%)$ \\
\hline \multirow[t]{4}{*}{ Age } & $22-31$ & 28 & 40 \\
\hline & $32-41$ & 25 & 35.7 \\
\hline & $42-51$ & 13 & 18.6 \\
\hline & $\geq 52$ & 4 & 5.7 \\
\hline \multirow[t]{3}{*}{ Educational level } & Diploma & 21 & 30 \\
\hline & Bachelor & 46 & 65.7 \\
\hline & Master & 3 & 4.3 \\
\hline \multirow[t]{2}{*}{ Nationality } & Saudi & 14 & 20 \\
\hline & Non-Saudi & 56 & 80 \\
\hline \multirow[t]{6}{*}{ Non-Saudi nationality } & Philippines & 24 & 34.3 \\
\hline & Malaysian & 20 & 28.6 \\
\hline & Indian & 8 & 11.4 \\
\hline & British & 1 & 1.4 \\
\hline & Solfican & 1 & 1.4 \\
\hline & Jordanian & 2 & 2.8 \\
\hline \multirow[t]{3}{*}{ Mother language } & Arabic & 16 & 22.9 \\
\hline & English & 1 & 1.4 \\
\hline & Other & 53 & 75.7 \\
\hline \multirow[t]{4}{*}{ Marital status } & Married & 39 & 55.7 \\
\hline & Divorced & 4 & 5.7 \\
\hline & Widow & 1 & 1.4 \\
\hline & Single & 26 & 37.1 \\
\hline \multirow[t]{4}{*}{ Number of children } & 0 & 36 & 51.4 \\
\hline & $1-2$ & 22 & 31.4 \\
\hline & $3-4$ & 10 & 14,3 \\
\hline & $5-6$ & 2 & 2.8 \\
\hline \multirow[t]{3}{*}{ Years of experience } & $2-10$ & 54 & 72.2 \\
\hline & $11-19$ & 12 & 17.1 \\
\hline & $\geq 20$ & 4 & 5,7 \\
\hline
\end{tabular}

Regarding awareness of the participants about herbal medicine use during pregnancy and breastfeeding, $88.6 \%$ of them heard about the term herbal medicine, $68.6 \%$ of them knew about its use by women during pregnancy and breastfeeding and $62.9 \%$ of them agreed that it is a branch of science. However, $91.4 \%$ of them did not attend any training or educational activity on herbal medicine and more than half of them were not aware of its benefits or harms (55.7\% \& $65.7 \%$ respectively) (Table 2$)$. 
Table 2: Awareness of herbal medicine use during pregnancy and breastfeeding of the participants.

\begin{tabular}{|c|c|c|c|c|}
\hline \multirow[t]{2}{*}{ Item } & \multicolumn{2}{|c|}{ Yes } & \multicolumn{2}{|r|}{ No } \\
\hline & Frequency & Percentage (\%) & Frequency & Percentage (\%) \\
\hline $\begin{array}{l}\text { Have you heard about the term } \\
\text { herbal medicine? }\end{array}$ & 62 & 88.6 & 8 & 11.4 \\
\hline $\begin{array}{l}\text { Did you attend a training or } \\
\text { educational activity on herbal } \\
\text { medicine? }\end{array}$ & 6 & 8.6 & 64 & 91.4 \\
\hline $\begin{array}{l}\text { Is herbal medicine a branch of } \\
\text { science? }\end{array}$ & 44 & 62.9 & 26 & 37.1 \\
\hline $\begin{array}{l}\text { Do you know about women use of } \\
\text { herbal products during pregnancy } \\
\text { and breastfeeding? }\end{array}$ & 48 & 68.6 & 22 & 31.4 \\
\hline $\begin{array}{l}\text { Are you aware with benefits of } \\
\text { herbal medicine use during } \\
\text { pregnancy and breastfeeding? }\end{array}$ & 31 & 44.3 & 39 & 55.7 \\
\hline $\begin{array}{l}\text { Are you aware with harmful effects } \\
\text { of herbal medicine use during } \\
\text { pregnancy and breastfeeding? }\end{array}$ & 24 & 34.3 & 46 & 65.7 \\
\hline
\end{tabular}

The reported benefits were increasing milk production (18.1\%), decreasing nausea and uterine contractions (1.4\%), promoting quick healing, relieving pain, and facilitating labour (4.6\%). While, the reported harmful effects were uterine hyper stimulation (11.4\%), birth defects, rashes, diarrhoea and vomiting for mother or baby (4.2\%), abortion and postpartum haemorrhage (5.6\%). Considering awareness of the participants regarding specific herbs, findings of the current study revealed that, $55.7 \%$ of them were familiar and aware of the effects of ginger $(55.7 \%)$ and garlic (51.4\%) Fig. 1. Additionally, less than half of them were familiar but unaware with the effects of basil (42.9\%), black seed $(37.1 \%)$ and Chamomile (34.3\%) Fig. 2. However, more than two thirds of them were unfamiliar to myrrh (78.6\%), fenugreek (71.4\%) and fennel (68.6\%) Fig. 3. Regarding the effects of specific herbs, $4.2 \%$ of them reported that cinnamon causes uterine hyper stimulation, fennel decrease heartburn and black seed increase immunity and decrease weight. 


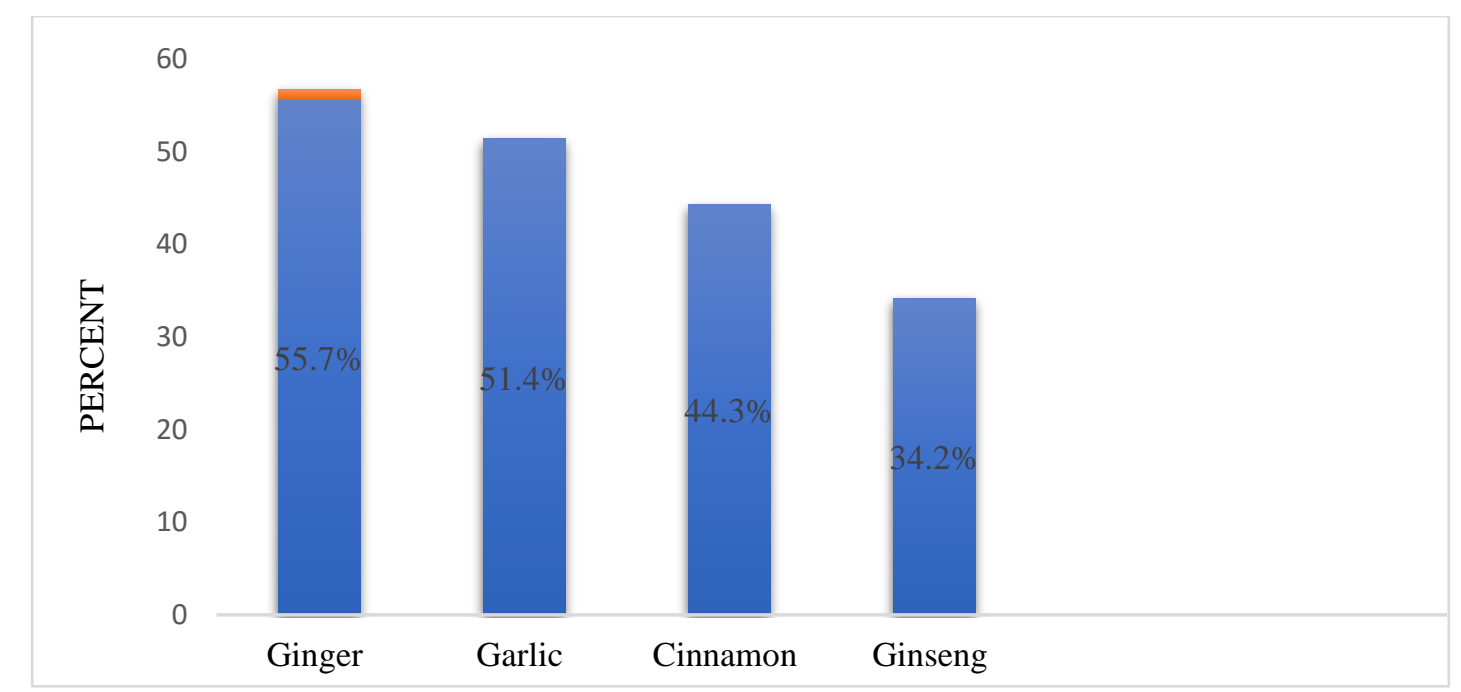

*Total is not exclusive

Figure 1: Common herbs used in pregnancy and breastfeeding nurses were familiar with and aware of their effects.

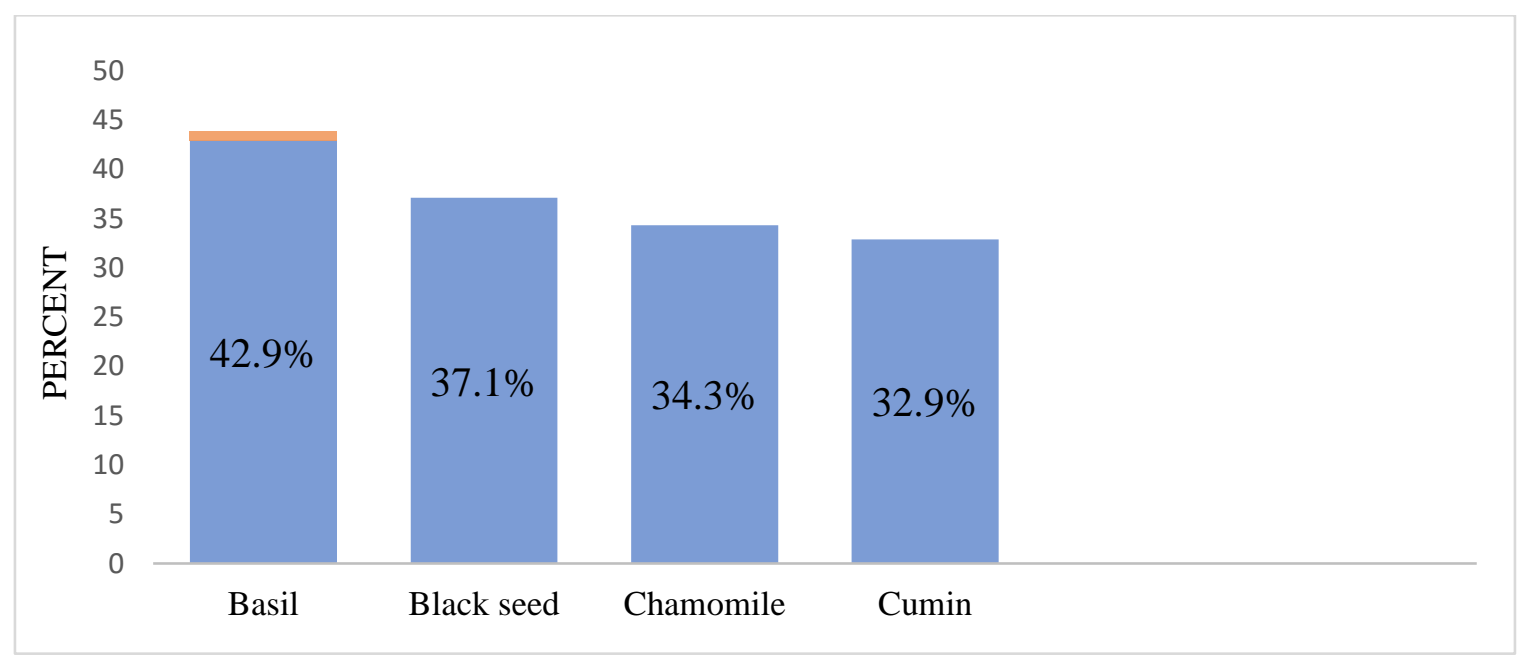

*Total is not exclusive

Figure 2: Common herbs used in pregnancy and breastfeeding nurses were familiar with but unaware of their effects. 


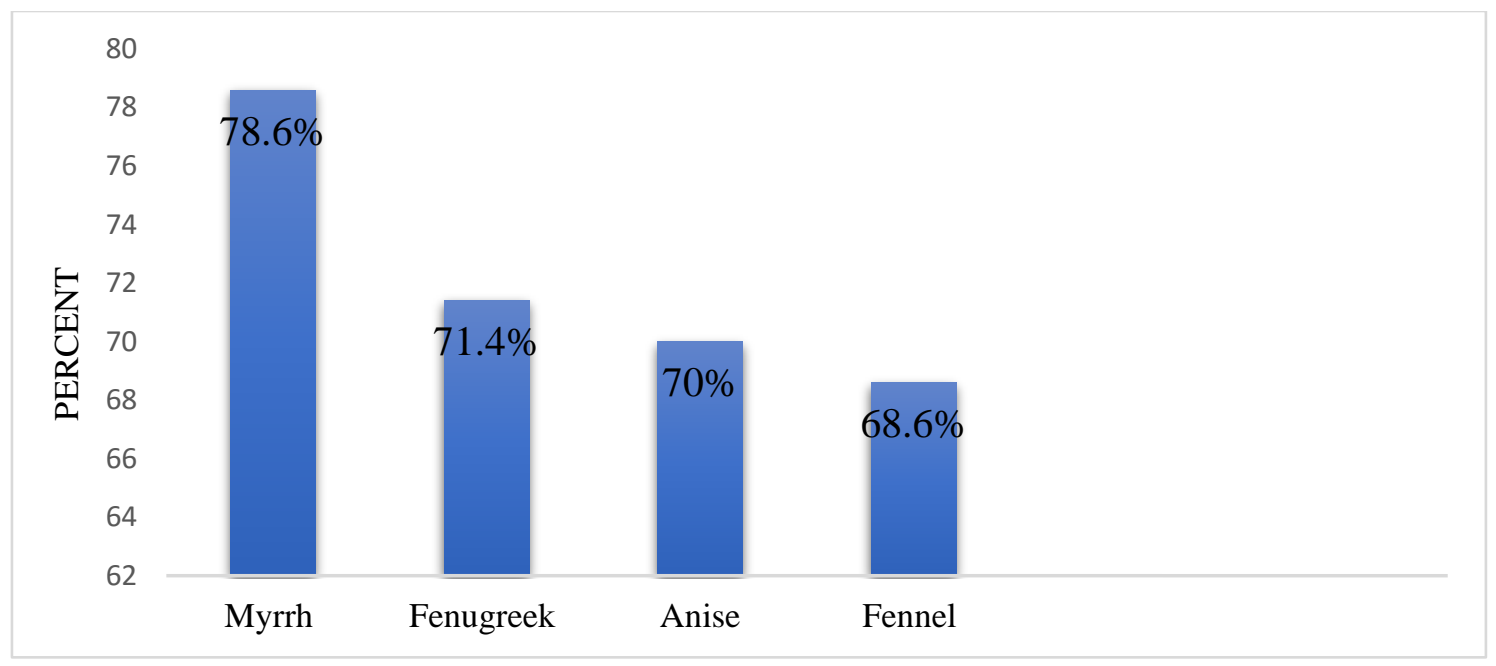

*Total is not exclusive

Figure 3: Common herbs nurses were unfamiliar with

Concerning attitudes of the participants towards herbal medicine use during pregnancy and breastfeeding, findings of the current study showed that, they had neutral attitudes (43\%) and positive attitudes (57\%). Statistically significant correlations were found between attitudes towards herbal medicine use and nurses' age in addition to number of children $(\mathrm{P}=0.03)$. Moreover, chi-square analysis revealed statistically significant relationships between nurses' nationality and their knowledge about women use of herbal products during pregnancy and breastfeeding $(\mathrm{P}=0.01)$ and their awareness with benefits of herbal medicine use during pregnancy and breastfeeding $(\mathrm{P}=0.03)$. Similarly, statistically significant relations were found between nurses' mother language and their knowledge about women use of herbal products during pregnancy and breastfeeding $(\mathrm{P}=0.01)$ and their awareness with benefits of herbal medicine use during pregnancy and breastfeeding $(\mathrm{P}=0.04)$.

\section{DISCUSSION}

The current study aimed at exploring awareness and attitudes of nurses towards herbal medicine use during pregnancy and breastfeeding in National Guard Hospital, Jeddah, KSA. Findings of the present study revealed that, more than three quarters of the participants have heard about the term herbal medicine. Additionally, more than half of them knew about women use during pregnancy and breastfeeding and agreed that herbal medicine is a branch of science. Meanwhile, more than half of them were not aware with the benefits or harms of using herbs during pregnancy and breastfeeding. The reason might be due to most of the participants did not attend any training or educational activity on herbal medicine. In contrast, previous study showed that, most of nurses neither agreed that herbal medicine is a branch of science nor knew some CAM practices (Şenay et al., 2015).

These findings can be supported by those revealed by other study conducted in Turkey regarding the midwives' knowledge and attitudes towards using herbal medicine during pregnancy and breastfeeding by Koc, et al. (2012) which indicated that, more than three quarters of the enrolled midwives have heard about herbal medicine. However, more than half of them were aware with the benefits of herbal medicine use during pregnancy and 
breastfeeding. In contrast, Şenay, et al. (2015) reported that, less than half of the included participants strongly agreed that herbal medicine is a branch of science although three quarters of them did not know some CAM practices. Furthermore, a previous study indicated that, more than three quarters of non-Muslim nurses working in Saudi Arabian obstetric units had no knowledge about the commonly used herbs by Muslim women (Sidumo, 2010). In the same context, findings of another study conducted in Riyadh revealed that most of the non-Saudi healthcare professionals had some knowledge regarding herbal medicine use (Abdullah, et al. 2012).

Current study showed that, half of the participants were familiar and aware with the effects of some herbs such as ginger and garlic and less than half of them were familiar but unaware with the effects of black seed and Chamomile. However, more than two thirds of them were unfamiliar to myrrh, fenugreek and fennel. In the same perspective, findings of a study conducted by Al-Rowais, et al. (2012) showed that, primary physician were familiar with honey and bee products (38.3\%), dietary supplements (34.9\%), garlic (47.1\%), ginger (41.9\%), cinnamon $(33.6 \%)$ and chamomile (26.6\%). In addition, they felt comfortable in counselling their patients about it. Moreover, Koc, et al. (2012) indicated that, nearly three quarters of involved midwives were suggesting some herbs use during pregnancy and breastfeeding as mulberry, dried figs and cassia for anaemia; nearly two thirds of them were suggesting mint, lemon and raspberry for nausea and vomiting; less than half of them were suggesting linden tea, sage, rose hip and fennel for the common cold and linden tea, rose hip and chamomile tea for sore throat.

Regarding the attitudes of nurses towards using herbal medicine during pregnancy and breastfeeding, current study findings indicated that, less than half of participants had neutral attitudes and more than half of them had positive attitudes towards it. Similarly, more than half of Turkish midwifes had positive attitudes and suggested herbal treatments for pregnant women. Additionally, more than three quarters of them agreed on the importance of informing pregnant women about use of herbal medicine (Koc, et al. 2012). In the same context, Samuel, et al. (2010) found in their study that many obstetricians had positive attitudes towards CAM, and some of them were using and recommending CAM to their pregnant women.

In the same perspective, findings of the present study showed that, nearly half of the participants agreed that herbal products can have side effects during pregnancy and breastfeeding. However, about one quarter of them agreed on recommending its use during pregnancy and breastfeeding. Accordingly, Koc, et al. (2012) found that, more than one quarter of the involved midwives agreed on recommending herbal medicine use to pregnant women because of being not harmful. However, more than three quarters of them confirmed that herbal medicine use during pregnancy may have fetal and maternal side effects.

Findings of the current study revealed a significant correlation between attitudes towards herbal medicine and nurses' age. Moreover, chi-square analysis revealed statistically significant relationships between nurses' nationality and their awareness of women use of herbal products during pregnancy and breastfeeding. These results are comparable to a previous study where no significant relationships were found between midwives' attitudes towards use of CAM and their sociodemographic background (Koc, et al. 2012). 


\section{CONCLUSIONS}

Based on the current study findings, it can be concluded that participating nurses knew about herbal medicine use by women during pregnancy and breastfeeding and had favourable attitudes towards it. Moreover, their attitudes were significantly correlated with their age and number of children. However, more than half of them need to be aware of benefits or harms of herbal medicine use during pregnancy and breastfeeding and did not attend any training or educational activity on herbal medicine. 


\section{REFERENCES}

Abdullah, M. A., Ahmed, T., El-Olemy, A. and Mohammed, K. M. (2012). Knowledge and attitude of health professionals in the Riyadh region, Saudi Arabia, towards complementary and alternative medicine. Journal of Family Community Medicine 19(2): 93-99. DOI: 10.4103/2230-8229.98290.

Abdullah, M., A, Ahmed, T., El-Olemy, A. and Mohammed, K. M. (2013). The use of and out-of-pocket spending on complementary and alternative medicine in Qassim province, Saudi Arabia. Annals of Saudi Medicine 33(3): 282-289. DOI: 10.5144/0256-4947.2013.282.

Adams, J. (2011). Growing popularity of complementary and alternative medicine during pregnancy and implications for healthcare providers. Expert Review of Obstetrics and Gynecology 6(4):365-366. DOI: 10.1586/eog.11.29.

AlBedah, M.N., El-Olemy, A. and Khalil, M. K. (2010). Knowledge, attitude, and practice of public in Riyadh region, Saudi Arabia, about complementary and alternative medicine. Egypt Journal of Community Medicine 28:3952. DOI: $10.4103 / 2230-8229.98290$

AlBedah, A.M., El-Olemy, A.T. and Khalil, M.K. (2012). Knowledge and attitude of health professionals in the Riyadh region, Saudi Arabia, towards complementary and alternative medicine. Journal of Family and Community Medicine 19(2): 93-99. DOI: 10.4103/2230-8229.98290.

Alrawi, S.N. and Fetters, M.D.( 2012). Traditional arabic and islamic medicine: a conceptual model for clinicians and researchers. Global Journal of Health Science 4(3):164-169. 10.5539/gjhs.v4n3p164.

Al-Rowais, N.A., Bedah, A.M., Khalil, M.K., el-Olemy, A.T., Khalil, A.A., Alrasheid, M. H. and Al-Khashan, H. (2012). Knowledge and Attitudes of Primary Health Care Physicians towards Complementary and Alternative Medicine in Riyadh Region, Saudi Arabia. Forschende Komplementarmedizin 19:7-12. DOI: $10.1159 / 000335814$.

Alzahrani, H., Bashawri, J., Salawati, E.M. and Bakarman, M.N. (2016). Knowledge and Attitudes towards Complementary and Alternative Medicine among Senior Medical Students in King Abdulaziz University, Saudi Arabia. Jeddah Saudi Arabia. Evidence-Based Complementary and Alternative Medicine. Hindawi Publishing Corporation. http://dx.doi.org/10.1155/2016/9370721.

Franco, P.F. (2016). Understanding Physicians' and Non-Physician Practitioners' Recommending Practices, Knowledge, Attitudes, Beliefs and Expectations Regarding Music as a Cost-Effective Complementary and Alternative Medicine Approach. Seton Hall University Dissertations and Theses (ETDs). Spring pp3-17 
Frawley, J., Adams, J., Sibbritt, D., Steel, A., Broom, A. and Gallois, C. (2013). Prevalence and determinants o f complementary and alternative medicine use during pregnancy: results from a nationally representative sample of Australian pregnant women. Austrilian and New Zealand Journal of Obstetrics and Gynaecology 53(4):347-52. DOI: 10.1111/ajo.12056

Frawley, J., Adams, J., Sibbritt, D., Steel, A., Broom, A. and Gallois, C. (2014). Majority of women are influenced by nonprofessional information sources when deciding to consult a complementary and alternative medicine practioner during pregnancy. Journal of Alternative and Compllementary Medicine 20(7): 571-7.DOI: 10.1089/acm.2014.0028

Fujiwara, K., Imanishi, J., Watanabe, S., Ozasa, K. and Sakurada, K. (2011). Changes in attitudes of Japnese doctors towards complementary and alternative medicine. Evidence-Based Complementary and Alternative Medicine. Hindawi Publishing Corporation. https://doi.org/10.1093/ecam/nep040

Hall, H., Giffiths, D. and Mckenna, L. (2011). The use of complementary and alternative medicines by pregnant women: a literature review. Midwifery 27(6)817-24. DOI: 10.1016/j.midw.2010.08.007.

Hall, H.R. and Jolly, K. (2014). Women's use of complementary and alternative medicines during pregnancy: a crosssectional study. Midwifery 30(5):499-505. DOI: 10.1016/j.midw.2013.06.001

Holst, L., Nordeng, H. and Haavik, S. (2008). Use of herbal drugs during early pregnancy in relation to maternal characteristics and pregnancy outcome. Pharmacoepidemiology and Drug Safety 17(2):151-159. DOI: $10.1002 /$ pds. 1527 .

Huang, N., Chou, Y.J., Chen, L.S., Lee, C.H., Wang, P.J. and Tsay, I.H. (2011). Utilization of western medicine and traditional Chinese nedicine services by physicians and their relatives: the role of training background. Evidence-Based Complementary and Alternative Medicine. Hindawi Publishing Corporation. https://doi.org/10.1093/ecam/nep094.

Kalder, M., Knoblauch, K., Hrgovic, I., and Munstedt, K. (2011). Use of complementary and alternative medicine during pregnancy and delivery. Arches of Gynecology and Obstetrics. 283(3):475-82. DOI: 10.1007/s00404010-1388-2

Koc, Z., Topatan, S., and Saglam, Z. (2012). Use of and attitudes towards complementary and alternative medicine among midwives in Turkey. European Journal of Obstetrics, Gynaecology and Reproductive Biology 160(2):131-136. DOI: 10.1016/j.ejogrb.2011.10.006. 
Lisha, J., J. and Nisha, S., N. (2015). Herbal Medicines Use During Pregnancy: A Review from the Middle East. Oman Medical Journal 30(4): 229-236. DOI: 10.5001/omj.2015.48

Mitchell, M. and Mcclean, S. (2014). Preganncy, risk perception and use of complementary and alternative medicine. Health Risk and Society Journal 16(1):101-16. DOI.org/10.1080/13698575.2013.867014

National Center for Complementary and Alternative Medicine (NCCAM). (2011). What Is CAM? Accessed on 2016 May 29. Available from: http://nccam.nih.gov/health/whatiscam/

Samuel, S.N., Zisk-Rony, R.Y. and Singer, S.R. (2010). Use of and attitudes towards complementary and alternative medicine among nurse-midwives in Israel. American Journal of Obstettics and Gynecology 203(4): 341.e17. DOI: 10.1016/j.ajog.2010.05.001.

Şenay, T.S., Gülzade, U.G. and Arzu, A.Y. (2015). Knowledge and Opinions of Nursing Students Regarding Complementary and Alternative Medicine for Cancer Patients. International Journal of Caring Sciences $8(3): 656-664$.

Sidumo, E.M., Ehlers, V.J. and Hattingh, S.P. (2010). Cultural knowledge of non-Muslim nurses' working in Saudi Arabian obstetric units. Curationis 33(3):48-55. DOI: 10.4102/curationis. v33i3.7.

Strouss, S., Mackely, A., Guillen, U., Paul, D. and Locke, R. (2014). Complementary and alternative medicine use in women during pregnancy: do their healthcare providers know? BMC Complementary and Alternative Medicine 14(85). DOI: 10.1186/1472-6882-14-85. 